\title{
A Novel Improvement for International Online Judging System and its Applications to Experimental Reform of C++ Course Yunping Zheng ${ }^{1, a,{ }^{*}}$, Mudar Sarem ${ }^{2, b}$, Qinghong Yang ${ }^{1, c}, X^{2}$ iaomeng Xie ${ }^{1, d}$
}

${ }^{1}$ School of Computer Science and Engineering, South China University of Technology, Guangzhou 510006, P. R. China

${ }^{2}$ School of Software Engineering, Huazhong University of Science and Technology, Wuhan 430074, P. R. China

azhengyp@scut.edu.cn, bmudar66@hotmail.com, ccsqhyang@scut.edu.cn, ${ }^{\mathrm{d}}$ xmxie@scut.edu.cn

Keywords: ACM/ICPC, C++, traditional experimental method, experimental reform, keywords, OJ, SCUTOJ.

\begin{abstract}
The ACM International Collegiate Programming Contest (ACM/ICPC) provides college students an opportunity to interact with students from other universities and to sharpen and demonstrate their problem-solving, programming, and teamwork skills. The $\mathrm{C}++$ course is an important professional backbone course for the students majoring in computer science. Given some shortcomings of the traditional experimental method of the $\mathrm{C}++$ course and the two vital and fatal shortcoming of the international Online Judging (OJ) system, in this paper, by adding two new parts to the description of the ACM/ICPC problem where some keywords either must appear or mustn't appear in the $\mathrm{C}++$ source codes, we first propose a novel improvement method for the international OJ system. Also, in this paper, we call our improved international OJ system as the SCUTOJ system since it is developed by South China University of Technology (SCUT). Then, we put forward a novel SCUTOJ-based experimental reform method for $\mathrm{C}++$ Course. Some detailed designing principles for the keywords and characteristics of the SCUTOJ-based exercises are presented. The proposed experimental reform method significantly improves the teaching quality and it is highly praised and generally welcomed by the students. Our SCUTOJ system overcomes the two vital and fatal shortcomings of the internal OJ system and can greatly decrease the overload of the teachers and simultaneously improve the efficiency of the teaching and the experiments of the $\mathrm{C}++$ course, and therefore it is a better system for contesting, teaching, and experiments of the $\mathrm{C}++$ course.
\end{abstract}

\section{Introduction of ACM/ICPC}

The ACM International Collegiate Programming Contest (ACM/ICPC) provides a platform for ACM, industry, and academia to encourage the public and focus their attentions on the next generation of computing professionals as they pursue excellence [1].

$\mathrm{C}++$ is a general purpose programming language based on the $\mathrm{C}$ programming language as specified in ISO/IEC 9899:1999 [2]. The course is very important for college students, especially those majoring in computer science. For the time being, the traditional experimental methods for the course mainly have the following three issues [3-6]:

Firstly, the students have no interests in a pure theory teaching method, and they generally feel that the $\mathrm{C}++$ course is difficult to learn and hard to apply.

Secondly, for the time being, the vast majority of colleges and universities put the great assessment of the $\mathrm{C}++$ course often on results, not on process in general.

Finally, the $\mathrm{C}++$ course, as a basic course in software design, can not culture the teamwork spirit in the traditional teaching method.

\section{International Online Judging System for C++ Course}

The international Online Judging (OJ) system is an online system for testing programs automatically. The program source code automatic online judging systems, often called Online Judge Systems or 
OJS for short, are widely used in many types of programming contests such as ACM/ICPC, Google Code Jam, TopCoder, etc. The system will feed back the correctness and the efficiency of the user codes [9]. The oldest and most recognized OJ system for programming contests was developed by Ciriaco Garcia de Celis in 1995 [10]. Some detailed design and implementation of the OJ can be found in [11-13].

For the time being, some colleges and universities have applied the ACM method to software foundation courses, such as high-level programming language C++ [3], data structure [7], and design and analysis of algorithms [8], and they have achieved remarkable teaching effects. However, vital and fatal shortcomings appear when we directly use the OJ system to design the problems of $\mathrm{C}++$. It is known that each problem in the international OJ system includes the seven parts. At first, we design the ACM/ICPC-based problems for the $\mathrm{C}++$ course by using the seven parts [3], however, it is unfortunate that some students seem to have found out the following two vital and fatal shortcomings of the internal OJ system:

The first shortcoming is that the internal OJ system can not check out whether the source codes embody some $\mathrm{C}++$ knowledge points which are required by the teacher or not. For example, if we want to solve a problem of computing the area of a circle, we can solve it by using the object-oriented or the procedure-oriented programming design method. However, the internal OJ system does have no knowledge of which kind of programming design method is used when the problem is correctly solved. Therefore, with the existence of this shortcoming, some students may submit their procedure-oriented source codes whereas object-oriented source codes are required in $\mathrm{C}++$ course, and vice versa.

The second shortcoming is that the internal OJ system can accept any source codes which can be written in different programming languages, such as Java, and $\mathrm{C} / \mathrm{C}++$. Therefore, taking advantage of this shortcoming, some students may not submit their source codes for the course exercises in $\mathrm{C}++$ language. However, when a student correctly submit his or her source code to the system in some languages other than $\mathrm{C}++$, the system can still accept his or her source code. Therefore, the teacher must check each student' source code to see whether his or her codes are written in C++ language, even if the student claims that he or she has correctly finished his course exercise for $\mathrm{C}++$.

\section{Our Proposed SCUTOJ-Based Experimental Reform for C++ Course}

In this section, we first propose a novel improvement method for the international OJ system. Also, in this paper, we call the improved international OJ system as the SCUTOJ system since it is developed by South China University of Technology. The website of our system can be linked by the web address: http://www.scut.edu.cn/oj/. Then, we put forward a novel SCUTOJ-based experimental reform method for $\mathrm{C}++$ Course.

A Novel Improvement for International OJ System. Considering the previous two shortcomings of the international OJ system for $\mathrm{C}++$ Course, in this paper, by adding two new parts which are described in the following paragraph to the description of the ACM/ICPC problem codes, we propose a novel improvement method for the international OJ system.

Now, by adding two new parts, i.e. KeyWord(s) that Must be Used and KeyWord(s) that Mustn't be Used, to the international OJ system, each problem in our SCUTOJ system includes the following nine parts:

- Description: This part is used to describe the problem to be solved and it can not be omitted.

- Input: This part is used to describe the input information of the algorithm and it can not be omitted.

- Output: This part is used to describe the output information of the algorithm and it can not be omitted.

- Sample Input: This part is used to describe some sample input information and it can not be omitted.

- Sample Output: This part is used to describe some sample output information and it can not be omitted. 
- KeyWord(s) that Must be Used: This part is used to confine some keywords that must appear in the source codes submitted by the programmer. In order to keep the consistent format and style of the problem as that of the international OJ system, this part can also be omitted when there is no need to confine the source codes.

- KeyWord(s) that Mustn't be Used: This part is used to confine some keywords that mustn't appear in the source codes submitted by the programmer. In order to keep the consistent format and style of the problem as that of the international OJ system, this part can also be omitted when there is no need to confine the source codes.

- HINT: This part is used to give some hint for the problem and it can be omitted.

- Source: This part is used to denote where the problem comes from and it can be omitted.

Our Proposed SCUTOJ-Based Experimental Reform Measures and Innovation for C++ Course. Our general principle for the experimental problems is as following: By strictly complying with the format of the SCUTOJ, we provide 6 exercises per chapter that must be solved by using the $\mathrm{C}++$ knowledge points required by the teacher. The students should complete at least four exercises per chapter.

The SCUTOJ-based experimental reform innovation can be described as follows:

Firstly, our SCUTOJ system can check out whether the source codes embody some C++ knowledge points, such as data types, classes, templates, exceptions, namespaces, operator overloading, function name overloading, references, and free store management operators, which are required by the teacher or not.

Secondly, our SCUTOJ system can accept only the C++ language by setting two new added parts with some specific keywords. For example, if the part of "KeyWord(s) that Must be Used" is used with keywords such as "cout", our SCUTOJ system will only accept the source codes written in C++ language since all the algorithms must have an output. Therefore, as far as our SCUTOJ system is concerned, the teacher doesn't need to check each student' source code to see whether his or her codes are written in $\mathrm{C}++$ language or not as our system can automatically judge whether the student has correctly finished his or her course exercise for the $\mathrm{C}++$ course or not. Therefore, our system can greatly decrease the overload of the teachers and simultaneously improve the efficiency of the teaching and the experiments of the $\mathrm{C}++$ course.

Thirdly, the knowledge point of each chapter was added into the exercises or the homework. Therefore, the students not only master the knowledge of each chapter, but they also understand the format and the style of the ACM/ICPC by submitting their program to our SCUTOJ system.

Finally, the SCUTOJ-based experimental course can be done through the network independently of time and space constraints. Different from the submitted assignments with document files or papers in the traditional experimental course, the students must write not only programs without compilation or logic errors, but also the input and the output formats of their programs must comply with the format of the SCUTOJ in order to be correctly accepted by our SCUTOJ system.

\section{Conclusions and Future Work}

In this paper, we propose a novel improvement method for the international OJ system and present our SCUTOJ system. Our SCUTOJ system overcomes the two vital and fatal shortcomings of the internal OJ system and it can greatly decrease the overload of the teachers and simultaneously improve the efficiency of the teaching and the experiments of the $\mathrm{C}++$ course, and therefore it is a better system for contesting, teaching, and experiments of the $\mathrm{C}++$ course. In the near future, we will design and develop an SCUTOJ-based examination system used for the final exam of the $\mathrm{C}++$ course.

\section{Acknowledgment}

This work is supported by the National Natural Science Foundation of China under Grant No. 61300134, the Natural Science Foundation of Guangdong Province of China under Grant No. 
S2013010012515, and No. 2015A030313206, the Fundamental Research Funds for the Central Universities of China under Grant No. 2015ZM133, the first batch's exploratory experimental teaching project of South China University of Technology under Grant No. x2js Y1140370, the teaching reform project of South China University of Technology under Grant No. x2js Y1141540, Guangdong higher school teaching quality and teaching reform project under Grant No. x2jsN9130800, the major teaching research project of South China University of Technology under Grant No. x2js Y1120020, and the third batch's exploratory experimental teaching project of South China University of Technology under Grant No. x2js Y1160280.

\section{References}

[1] http://icpc.baylor.edu/worldfinals/rules, 2016.

[2] http://www.open-std.org/JTC1/SC22/WG21/docs/standards\#14882, 2016.

[3] Yunping Zheng A novel method of reform and exploration of $\mathrm{C}++$ bilingual teaching based on ACM/ICPC In: Proceedings of 3rd International Conference on Information, Electronic and Computer Science, 2011, 626-630.

[4] Wang. Chunling Practical teaching reform for $\mathrm{C}++$ course program design. Computer education, 2007, 12: 3-4.

[5] He Wenxiao, Zhong Qi. The reform of experiment teaching for the c++ language program design. Modern computer, 2009, 7: 84-86.

[6] Liu Xiaoyan, Hu Yanling. Teaching reform and research based on the object-oriented c++ programming. Computer education, 2007, 1: 877-878.

[7] $\mathrm{Wu}$ Jianhua. Data structure teaching reform and exploration based on the pattern of ACM. Computer education, 2007, 12: 114-116.

[8] Yunping Zheng, Sarem Mudar. A New ACM/ICPC-Based Teaching Reform and Exploration of "Design and Analysis of Algorithms". Lecture Notes in Electrical Engineering, 2013, 269(1): 123-131.

[9] Cheedoong Drung, Jianwen Wang, Ning Guo. Enhance performance of program automatic online judging systems using affinity algorithm and queuing theory in SMP environment. In: Proceedings of International Conference on Electronic \& Mechanical Engineering and Information Technology, 2011, 9:4425- 4428.

[10] M. Revilla, S. Manzoor, R. J. Liu. "Competitive Learning in Informatics: The UVa Online Judge Experience," Olympiads in Informatics, vol. 2, pp. 131-148, 2008.

[11]J. W. Tang. "Summarization of ACM/ICPC Online Judge System," Computing Technology and Automation, vol. 24, Dec. 2005, pp. 405-409.

[12]H. Wang, X. H. Hu and G. Q. Zhang. "The Design and Exploitation of Cluster-based Judging System for Programming Contest," Computer Applications and Software, vol. 26, Sep. 2009, pp. 119-122.

[13] M. Meulen and M. Revilla, "Experiences with the Design of a RunTime Check," Proc. 24th Int'l Conf. Computer Safety, Reliability and Security, J. Gorski, ed., pp. 302-315, 2006. 